\title{
Differential Diagnosis of Post-Procedural Skin Lesions: A Case Report
}

\author{
Taruna Waghray-Penmetcha, Sam Li, Rohit Puranik, Maria L. Torres \\ John H. Stroger Jr. Hospital of Cook County, Chicago, IL, USA \\ Email: tpenmetcha@cookcountyhhs.org, twaghray21@gmail.com, sli2@cookcountyhhs.org, \\ rpuranik@gmail.com, mtorres4@cookcountyhhs.org
}

Received 4 September 2015; accepted 15 January 2016; published 18 January 2016

Copyright @ 2016 by authors and Scientific Research Publishing Inc.

This work is licensed under the Creative Commons Attribution International License (CC BY).

http://creativecommons.org/licenses/by/4.0/

(c) (7)

Open Access

\begin{abstract}
Skin reactions caused by interventional pain procedures are well documented in literature, ranging from fistula formation to urticarial allergic reactions and infections. Burn lesions may also occur, however far less common; and as pain physician we must be cognizant of this possible complication and its etiologies. This is difficult in an outpatient setting where a patient cannot be regularly monitored, their adherence to prescribed therapies is unclear, and reporting is often done via phone, ancillary staff, and outside facility records. These compounding factors require clinicians to consider a broad differential and be comfortable with instituting myriad therapies or appropriately involve outside consultation for thorough patient care.
\end{abstract}

\section{Keywords}

Facet Arthropathy, Skin Lesions, Transcutaneous Electrical Stimulation, Capsaicin

\section{Introduction}

Low back pain is the most prevalent cause of pain, lost work time and disability. Degenerative diseases of the spine such as spondylosis, facet arthropathy and degenerative disc disease contribute to a large number of cases of back pain [1]. Facet joints or zygapophyseal joints are a pair of joints in the posterior aspect of the spine. These are true synovial joints created as the inferior articular process of the vertebral body above meets the lower superior articular process. Lumbar facet joints have been implicated as a cause for chronic low back pain in $15 \%$ - $45 \%$ of the patients with back pain. Diagnostic facet blocks or Medial branch blocks with local anesthetic with or without steroids are the only reliable way to diagnose facet joint pain [2].

Spine interventions can result in complications like infection, bleeding, nerve damage etc. Skin reactions caused by interventional pain procedures are also well documented, ranging from fistula formation to urticarial 
allergic reactions. Burn lesions do occur, however far less common; and as pain physician we must be cognizant of this possible complication and its etiologies.

\section{Case Description}

We are presenting a case of a 45-year-old African American female, who was referred to our pain clinic for management of chronic low back pain. Her past medical history was significant for obesity, depression, tension headaches, tinea pedis, and aspirin allergy. Her home medications included Miconazole ointment for tinea pedis, Citalopram and Venlafaxine for depression, Diclofenac, Capsaicin Cream, Lidoderm Patches, and TENS unit for back pain. She complained of low back pain that had gotten worse over past 2 years. Pain was described as sharp, aching pain in the lower back, worse with walking and going down the stairs. Physical exam showed positive facet loading and pain with extension. MRI showed degenerative disc disease and facet arthropathy at L3-S1 with an old left L1 transverse process fracture. Based on her history, exam and MRI, she was diagnosed with lumbar facet arthropathy. The patient underwent bilateral lumbar medial branch nerve blocks of the L3/4, L4/5 and the ala of sacrum. She was prepped with chlorhexidine and draped in a sterile fashion. Lidocaine $1 \%$ was used to localize the skin. She received a total of 6 separate injections containing bupivacaine $0.25 \%$ with dexamethasone at each level (total 4 cc bupivacaine with $8 \mathrm{mg}$ of dexamethasone). All the medications were obtained from the pyxis and drawn in sterile fashion. No immediate complications were seen. Post-procedure day 4, the patient called the clinic with complaint of drainage of purulent material adjacent to previous injections sites, but denied any fever or chills. Patient was advised to come to the clinic but she chose to go to emergency room. At the ER visit, she was prescribed a course of oral antibiotics. She then self-administered Capsaicin and Triamcinolone cream to the affected area. Subsequent examination revealed a healing superficial skin ulcer, $3 \times$ $4 \mathrm{~cm}$, located on left buttock, distant from the injection site. Burn service was consulted, lesion was considered a $2^{\text {nd }}$ degree skin burn and wound care regimen was prescribed. Pain was managed with pharmacological therapy (Figure 1).

- Post-Procedure Day 0: No immediate complications were noted.

- Post-Procedure Day 4: Pt. called the clinic complaining of drainage of purulent material adjacent to previous injections sites, but denied any fever or chills. Patient told to come to the Pain clinic but went to ED, given PO antibiotics and local antibiotic cream.

- Post-Procedural Day 14: She had completed a course of oral antibiotics, while, self administering Capsaicin and Triamcinolone cream to the affected area.

- Post procedure Day 34: Patient came to the clinic for follow up. Left buttock healing superficial skin ulcer, 3 $\times 4 \mathrm{~cm}$, located on left buttock, distant from the injection site. Burn service was consulted, lesion was considered a $2^{\text {nd }}$ degree skin burn and wound care regimen was prescribed. Her CLBP was medically managed henceforth.

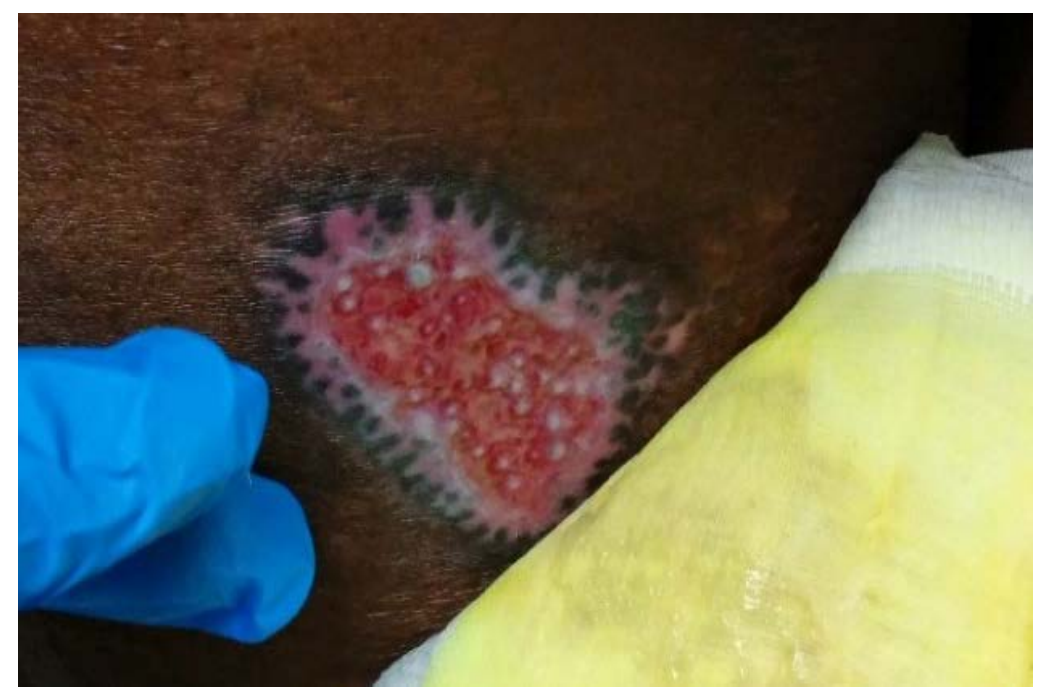

Figure 1. Skin burn. 
Table 1. Skin lesions after procedure.

\begin{tabular}{|c|c|c|}
\hline Likely Etiology & Literature Support & Comments \\
\hline Infection & Systemic signs: fever, elevated white count. & $\begin{array}{l}\text { Given the distant site from the injection } \\
\text { and lack of systemic signs and symptoms, } \\
\text { infection seemed unlikely }\end{array}$ \\
\hline $\begin{array}{l}\text { Allergic reaction } \\
\text { (to one or more agents) }\end{array}$ & $\begin{array}{l}\text { Symptoms may range from blanching of the skin to urticarial } \\
\text { lesions along the distribution of placement. }\end{array}$ & Prep, drape, tape, or bandages \\
\hline Steroid use & $\begin{array}{l}\text { Acute generalized exanthematous pustulosis } 2 \text { days after SQ } \\
\text { injection of dexamethasone. }\end{array}$ & \\
\hline $\begin{array}{l}\text { Local irritation } \\
\text { (topical agents) }\end{array}$ & $\begin{array}{l}\text { Capsaicin causes local vasodilation and inhibits both } \\
\text { autonomic and somatic nerves. Capsaicin at concentration of } \\
\text { at least } 0.1 \% \text {, has shown to penetrate into a collagen mixture } \\
\text { and be cytotoxic to both keratinocytes and fibroblasts. }\end{array}$ & $\begin{array}{l}\text { Our patient used capsaicin cream } 0.125 \% \\
\text { on the open lesion. }\end{array}$ \\
\hline Heating pads & $\begin{array}{l}\text { Heating pad can result in a thermal burn. High risk in elderly } \\
\text { patients, and patients with sensory deficits, atrophic skin, } \\
\text { vascular insufficiency. }\end{array}$ & \\
\hline TENS & $\begin{array}{l}\text { Case reports of third degree burn from interferential current } \\
\text { therapy using the TENS machine and cutaneous irritation and } \\
\text { contact dermatitis from pad placement. }\end{array}$ & \\
\hline
\end{tabular}

\section{Discussion}

In regards to our patient, possible differential diagnoses of skin lesion include infection or reaction to one or more agents: prep/drape/tape/bandages, dexamethasone, capsaicin, heating pad, and TENS unit. Given the distant site from the injection and lack of systemic signs and symptoms, infection seemed unlikely. Many case reports state that contact dermatitis or chronic skin irritation can develop from various agents. Symptoms may range from blanching of the skin to urticarial lesions. Further, it is possible to develop acute generalized exanthematous pustulosis 2 days after SQ injection of dexamethasone [3]. Another study shows that a single massive dose of steroid did not influence abscess formation in patients inoculated with Staphylococcus aureus [4]. Capsaicin causes local vasodilatation and creates both autonomic and somatic nerve inhibition. Topical Capsaicin was mixed with cultures of human keratinocytes and human fibroblasts and incubated over time. At concentration of at least $0.1 \%$, capsaicin is shown to penetrate the inner layers of this collagen mixture and be cytotoxic to both keratinocytes and fibroblasts [5]. Combined use of capsaicin, and a heating pad can result in a thermal burn. There has been a case report of third degree burn from interferential current therapy using the TENS machine [6], other side effects may include cutaneous irritation and contact dermatitis from pad placement (Table 1).

\section{Conclusion}

As interventional pain management physicians, we will be exposed to procedure related complications including skin lesions. It can be tenuous to manage outpatient complications after pain procedures. Whether it is a lack of monitoring, patient noncompliance, or a lack of communication, these compounding factors require clinicians to consider a broad differential, be comfortable with alternative therapies, and appropriately involve other consultation services. In our case, although the etiology of her skin lesion was unclear, given the temporal relationship of the lesion to the procedure, we explored all possible differentials. We also consulted our burn/wound care service early, in addition to our continued care.

\section{References}

[1] Benzon, H.T., Raja, S.N., Molloy, R.E., Liu, S.P. and Fishman, S.M. (1999) Essentials of Pain Medicine and Regional Anesthesia. 2nd Edition, Elsevier, Amsterdam.

[2] Manchikanti, L., Singh, V., Falco, F.J.E., Cash, K.A. and Pampati, V. (2010) Evaluation of Lumbar Facet Joint Nerve Blocks in Managing Chronic Low Back Pain: A Randomized, Double-Blind, Controlled Trial with a 2-Year FollowUp. International Journal of Medical Sciences, 7, 124-135. http://dx.doi.org/10.7150/ijms.7.124

[3] Demitsu, T., Kosuge, A., Yamada, T., Usui, K., Katayama, H. and Yaoita, H. (1996) Acute Generalized Pustulosis In- 
duced by Dexamethasone Injection. Dermatology, 193, 56-58. http://dx.doi.org/10.1159/000246204

[4] Joiner, K.A., Gelfand, J.A., Bartlett, J.G. and Gorbach, S.L. (1981) The Effect of Corticosteroids on Subcutaneous Abscess Formation in the Mouse. British Journal of Experimental Pathology, 62, 222-226.

[5] Ko, F., Diaz, M., Smith, P., Emerson, E., Kim, Y.J., Krizek, T.J. and Robson, M.C. (1998) Toxic Effects of Capsaicin on Keratinocytes and Fibroblasts. Journal of Burn Care and Research, 19, 409-413. http://dx.doi.org/10.1097/00004630-199809000-00010

[6] Satter, E.K. (2008) Third-Degree Burns Incurred as a Result of Interferential Current Therapy. American Journal of Dermatopathology, 30, 281-283. http://dx.doi.org/10.1097/DAD.0b013e31816a9d4f 